\title{
Impact of topical oxygen therapy on diabetic foot ulcer healing rates: a systematic review
}

\author{
Objective: The aim of this systematic review was to determine \\ the impact of topical oxygen therapy (TOT) on diabetic foot ulcer \\ (DFU) healing. \\ Method: Using systematic review methodology, we considered \\ randomised controlled trials (RCTs), controlled trials, pilot studies and \\ observational studies. The search was conducted in January 2019, \\ using PubMed, CINAHL, Ovid, Cochrane, Web of Science and \\ EMBASE databases. Data analysis was undertaken using RevMan \\ and a narrative synthesis. The article titles were assessed by two \\ authors independently, and the abstracts (when available) of the \\ studies identified by the search strategy were screened for their \\ eligibility, according to the inclusion and exclusion criteria. The \\ full-text version of potentially relevant studies was obtained and two \\ authors independently screened this against the inclusion criteria. \\ Data were extracted using a predesigned extraction tool and all \\ included studies were quality appraised using the Evidence-Based \\ Librarianship checklist. \\ Results: The search returned 565 records of which eight met the \\ inclusion criteria. Of the included studies, three were set in single
}

\begin{abstract}
centre outpatient wound clinics, two studies were set in an outpatient wound care research clinic and three studies were multisite. Meta-analysis of four studies was undertaken. DFUs are $>2$ times more likely to heal with TOT than with standard care alone. The odds ratio $(\mathrm{OR})=2.49$ (95\% confidence interval $(\mathrm{Cl}): 1.59-3.90$, $\mathrm{p}=0.00001)$. The remaining four studies also showed that using TOT increased healing rates. An included study reported that time to $50 \%$ DFU closure was significantly shorter in participants who received the TOT, mean 18.4 days versus 28.9 days in the sham therapy group $(p=0.001)$. However, the validity of $65.5 \%$ of the eight studies was assessed as low.

Conclusion: The findings suggest that TOT enhances healing for patients with hard-to-heal DFUs when used with standard care. The results from the trials reviewed also indicate a benefit for patients over standard care alone. However, the sample sizes in the studies were generally small, thus, more RCTs are warranted to further validate these findings.

Declaration of interest: The authors have no conflicts of interest to declare.
\end{abstract}

evidence-based librarianship checklist $\bullet$ diabetes $\bullet$ diabetic foot ulcer $\bullet$ randomised controlled trial $\bullet$ standard care $\bullet$ systematic review $\bullet$ topical oxygen therapy $\bullet$ treatment $\bullet$ wound $\bullet$ wound care $\bullet$ wound healing $\bullet$ wounds

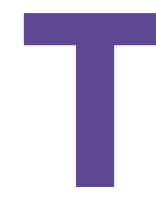

he International Working Group on the Diabetic Foot defines a foot ulcer as a full thickness wound involving the foot or the ankle. ${ }^{1}$ As a common complication of diabetes, diabetic foot ulcers (DFUs) can lead to a significant psychological, social and economic burden on both the health system and the patient. ${ }^{2}$

A study in 2005 by Boulton et al. ${ }^{3}$ estimated that, globally, every 30 seconds a limb was lost as a result of diabetes and that a person with diabetes had a lifetime risk of developing a foot ulcer of $25 \%$. A further study carried out in 2011 by Bakker et al. ${ }^{4}$ showed that this figure had increased to a limb lost every 20 seconds from complications of diabetes. A study carried out over a five-year period from 2005 and 2009 showed that in Ireland lower extremity amputations related to diabetes were between $145-176$ per 100,000 persons, and a person with diabetes was 22.3 times more likely to have a lower limb amputation than those without diabetes. 5

DFUs are a substantial and mounting problem. It has been estimated that one in every four people with diabetes is at risk of developing a DFU. ${ }^{6}$ In 2017 it was estimated that 451 million adults worldwide had diabetes, with this figure expected to increase to 693 million by $2045 .{ }^{6}$ In Europe, the figure was estimated to be 59.8 million, which equated to $9.1 \%$ of the population aged between 20 and 79 years. ${ }^{7}$ In the UK, diabetes has been estimated to affect approximately 3.8 million people. ${ }^{8}$

An Irish study in 2014 found that wound care accounts for $5 \%$ of the active caseload for community

Frances Connaghan, ${ }^{1}$ Tissue Viability Nurse; Pinar Avsar, ${ }^{2} \mathrm{Dr}, \mathrm{PhD}, \mathrm{MSc}, \mathrm{RGN}$, Senior Postdoctoral Fellow*; Declan Patton, 2,3,4,5 Professor, PhD, MSc, PGDipEd, PGCRM, BNS(Hons), RNT, RPN, Professor of Nursing and Midwifery Research, Deputy Director; Tom O'Connor, 2,5,6,7 Professor, EdD, MSc Ad Nursing, PG Dip Ed, BSc, Dip Nur, RNT, RGN, Director of Academic Affairs and Deputy Head of School, School of Nursing and Midwifery and Lead Researcher; Zena Moore, ${ }^{2,5,6,7,8,9}$ Professor, PhD, MSc (Leadership in Health Professionals Education), MSc (Wound Healing \& Tissue Repair), FFNMRCSI, PGDip, Dip First Line Management, RGN, Professor of Nursing, Head of School of Nursing and Midwifery, Director

*Corresponding author email: pinaravsar@rcsi.com

1 Cavan \& Monaghan Primary Care Services, HSE CHO 1, Ireland. 2 Skin Wounds and Trauma Research Centre, Royal College of Surgeons in Ireland, University of Medicine and Health Sciences, Dublin, Ireland. 3 Adjunct Associate Professor, Fakeeh College of Health Sciences, Jeddah, Saudi Arabia. 4 Honorary Senior Fellow, Faculty of Science, Medicine and Health, University of Wollongong, Australia. 5 Adjunct Professor, Griffith University, Australia. 6 Honorary Professor, Lida Institute, Shanghai, China. 7 Adjunct Professor, Fakeeh College of Health Sciences, Jeddah, Saudi Arabia. 8 Professor, Department of Public Health, Faculty of Medicine and Health Sciences, Ghent University, Belgium. 9 Visiting Professor, University of Wales, Cardiff, UK. 
nurses, with $1 \%$ of patients being treated for a DFU. ${ }^{9} \mathrm{~A}$ study carried out to estimate the cost of treating a DFU in an Irish hospital setting estimated it to be $€ 23,489$ per hospital admission and the authors stated this was an underestimation as it did not include costs for blood testing or antibiotic therapy. ${ }^{2}$

Many DFUs occur secondary to peripheral vascular disease, ${ }^{10}$ leading the tissue to be in a chronic state of hypoxia; restoring oxygen to the wound bed would therefore seem like a logical step in wound treatment. This can be done through revascularisation surgery; however, many patients with DFUs have comorbidities making them unsuitable candidates for surgery, thus, an alternative treatment of topical oxygen delivery systems may need to be used.

Topical oxygen therapy (TOT) is defined as the administration of oxygen applied topically over injured tissue by either continuous delivery or pressurised systems. ${ }^{11}$ Topical continuous oxygen is also known as continuous diffusion of oxygen (CDO) and provides a flow of oxygen to the wound bed, usually at a rate of $3-12 \mathrm{ml} /$ hour. The oxygen is delivered through a portable device. Topical pressurised oxygen therapy was originally termed topical hyperbaric oxygen therapy and has been in use since the 1960s. It works by placing a bag, boot or extremity chamber around the affected area. It is sealed tightly to prevent leaks and oxygen is delivered, usually from an outside tank, at a rate of $5-601 /$ minute to pressures just above that of atmospheric pressure. ${ }^{12}$ It is not continuous and sessions usually last 90 minutes/day, for 3-5 days/week but sometimes more. Gottrup et al. ${ }^{11}$ have been able to d raw $\quad$ on $\quad$ s y s tem a t ic research into wound healing. The authors also focused on topical and hyperbaric oxygen treatment in their search.

There is, at the time of writing, no systematic review that evaluates the use of TOT for DFUs. Therefore, this systematic review aimed to synthesise the published literature to determine the impact of TOT on healing rates among patients with DFUs.

\section{Methods}

\section{Research question}

The research question was as follows: 'What is the impact of TOT on healing rates among patients with DFUs?'

\section{Criteria for considering studies for this review}

Types of studies: the systematic review included published and unpublished randomised controlled trials (RCTs), controlled trials, pilot studies and observational studies. Case series papers were excluded. There were no restrictions with respect to date of publication or trial setting. The only restriction to the search was that studies were published in the English language.

Types of participants: any adult only with DFUs.
Types of interventions: studies describing the following comparison were eligible for the review: TOT compared with no TOT.

\section{Types of outcome measures}

Primary outcome: wound healing, which could have been reported as the proportion of patients with DFUs that have reduced wound circumference or complete wound closure following the intervention.

Secondary outcomes: rate of healing, recurrence rates, pain and adverse reactions.

\section{Electronic searches}

The following electronic databases were searched to identify relevant literature:

- The Cochrane Central Register of Controlled Trials (CENTRAL)

- The Cochrane library (latest issue)

- Ovid

- Cumulative CINAHL Plus (Cumulative Index to Nursing and Allied Health Literature).

- PubMed

- Web of Science

- EMBASE.

Assistance from an expert librarian was sought to help develop and identify appropriate keywords to use.

\section{Searching other resources}

The bibliographies of all retrieved publications were searched to identify any further studies. The author of a study ${ }^{13}$ that had only produced interim results was contacted for clarification on some issues. Dressing manufacturers were contacted to enquire about current trials or recently completed trials. Ongoing clinical trials registers were also searched:

- Clinicaltrials.gov (www.clinicaltrials.gov)

- World Health Organization International Clinical Trials Registry Platform (WHO ICTRP)

- EU Clinical Trials Register ( www.clinicaltrialsregister. eu).

For studies that met the inclusion criteria, the listed contact person was emailed to seek any available results of the study.

\section{Study selection}

The article titles were assessed by two authors independent of each other, and the abstracts (when available) identified by the search strategy were screened for their eligibility, according to the inclusion and exclusion criteria. The full-text version of potentially relevant studies was obtained and two authors independently screened this against the inclusion criteria. Consensus between the two authors in relation to the studies and the data to be included was obtained through a discussion when discrepancies were identified. 


\section{Data extraction}

Data from the retrieved articles were extracted and inserted into a table using the following headings: study name, author, date of study, setting, unit, sample size, design, intervention, comparison and outcomes.

\section{Quality appraisal and data analysis}

The quality of studies was assessed independently by two authors, without blinding to journal or authorship, using The evidence-based librarianship (EBL) quality appraisal. ${ }^{14}$ This quality appraisal tool assesses the validity, the applicability and appropriateness of a study, based on four main steps of the research process:

- Population

- Data collection

- Study design

- Results.

According to this checklist, if the overall validity of the study (Yes/Total) is $\geq 75 \%$ or ((No+Unclear)/Total) is $\leq 25 \%$, then the study is valid. ${ }^{14}$

In this review, following the extraction of the main findings from the papers, meta-analysis statistical synthesis was undertaken using RevMan. ${ }^{15}$ Relative risks (RR) and 95\% confidence intervals (CI) were calculated for dichotomous outcomes. Results of comparable trials were pooled using the fixed-effect model and 95\% CI for the meta-analysis. Heterogeneity was investigated by calculating the I 2 statistic. ${ }^{16}$

\section{Results}

\section{Overview of all included studies}

Fig 1 outlines the flow of articles through this review. After the initial search, a total of 563 hits were achieved, and following assessment, 16 articles were excluded as they did not meet the eligibility criteria (Fig 1).

The authors of two papers were contacted; one ${ }^{17}$ was a retrospective chart review of hard-to-heal wound patients treated with TOT carried out in the US. The author was contacted to see if data extracted on wound location had specified if any of the wounds were DFUs. The author responded that they had not collected this data, and so this paper was excluded. Another author ${ }^{13}$ of a multicentre RCT which had only published interim results was contacted to clarify some issues. Following correspondence from the author, it was decided to include this trial as it had produced results from the 12-week mark.

Finally, eight papers ${ }^{10,13,18-23}$ met the inclusion criteria and these papers formed the basis of this review.

\section{Study design}

Of the eight studies, five were RCTs, ${ }^{10,13,20,21,23}$ one was a prospective controlled study, ${ }^{18}$ one was a pilot study, ${ }^{22}$ and one was a retrospective, non-comparative observational study. ${ }^{19}$

\section{Geographical location}

The geographical location of the studies varied between Canada, ${ }^{10,18,19}$ the US, ${ }^{20,21,23}$ and a multinational study conducted in the $\mathrm{UK}^{22}$, US, France, Germany and Luxembourg. ${ }^{13}$

\section{Study settings}

A single centre outpatient wound clinic was the setting for three studies, ${ }^{10,18,19}$ two studies were set in an outpatient wound care research clinic, ${ }^{20,23}$ and three studies, ${ }^{13,21,22}$ were multisite.

\section{Sample size}

The mean sample size was 47 participants (standard deviation $(\mathrm{SD})=41.9)$ varying between 10 participants $^{22}$ and 124 participants. $^{23}$

Table 1. Quality appraisal results

\begin{tabular}{|c|c|c|c|c|c|}
\hline Authors & $\begin{array}{l}\text { Population } \\
\text { category (\%) }\end{array}$ & $\begin{array}{l}\text { Data collection } \\
\text { category }(\%)\end{array}$ & $\begin{array}{l}\text { Study design } \\
\text { category }(\%)\end{array}$ & $\begin{array}{l}\text { Results category } \\
(\%)\end{array}$ & $\begin{array}{l}\text { Overall validity } \\
\text { (\%) }\end{array}$ \\
\hline \multirow[t]{2}{*}{ Blackman et al., $2010^{18}$} & 62.5 & 80.0 & 80.0 & 100.0 & 69.5 \\
\hline & Not valid & Valid & Valid & Valid & Not valid \\
\hline \multirow[t]{2}{*}{ Kemp and Hermans, $2011^{19}$} & 0.0 & 0.0 & 0.0 & 0.0 & 0.0 \\
\hline & Not valid & Not valid & Not valid & Not valid & Not valid \\
\hline Driver et al., $2013^{20}$ & 62.5 & 37.5 & 100.0 & 50.0 & 59.0 \\
\hline \multirow[t]{2}{*}{ Yu et al., $2016^{10}$} & 75.0 & 80.0 & 80.0 & 80.0 & 78.0 \\
\hline & Valid & Valid & Valid & Valid & Valid \\
\hline \multirow[t]{2}{*}{ Niederauer et al., $2017^{21}$} & 87.5 & 100.0 & 80.0 & 100.0 & 92.0 \\
\hline & Valid & Valid & Valid & Valid & Valid \\
\hline Hayes et al., $2017^{22}$ & 40.0 & 60.0 & 100.0 & 83.0 & 71.0 \\
\hline \multirow{2}{*}{ Frykberg et al., $2019^{13}$} & 85.0 & 83.0 & 80.0 & 17.0 & 66.0 \\
\hline & Valid & Valid & Valid & Not valid & Not valid \\
\hline
\end{tabular}




\section{Primary outcomes}

Wound healing: proportion of patients with DFUs that have reduced wound circumference or complete wound closure following the intervention.

A meta-analysis of five RCTs, ${ }^{10,13,18,21,23}$ was undertaken. The results are presented in a forest plot (Fig 2). Of 177 participants with DFUs who received TOT, $53.6 \%(n=95)$ had full wound closure. In the group of 172 participants who received standard care, 31.3\% $(n=54)$ had full wound closure. Results of the analysis show an odds ratio of healing of 2.49 (95\% CI: 1.593.90; $\mathrm{p}=0.00001)$. From this, we can see that DFUs are $>2$ times more likely to heal with TOT than with standard care alone. It should be noted for the purpose of this review that we did not differentiate the methods of TOT used while carrying out this analysis. Due to the small number of studies retrieved, it was decided to look at the intervention of topical oxygen as a whole group.

\section{Secondary outcomes}

Healing rates: rate of healing in the study by Blackman et al. ${ }^{18}$ was measured as median time to closure. In the therapy group this was 56 days (interquartile range (IQR): 39-81 days) in comparison with 93 days (IQR: 62-127 days) in the control group. The estimate of time to complete closure using Kaplan-Meier was: therapy group=94 days, control group=340 days $(\mathrm{p}=0.013)$.

Kemp and Hermans ${ }^{19}$ showed a median time to closure of 46 days (range: 13-119 days) for the 12 ulcers that healed in their study. Previously, the ulcers had an average duration of 134 days (range: 14-350 days). Niederauer et al. ${ }^{21}$ reported that time to 50\% DFU closure was significantly shorter in participants who received the CDO therapy than in the sham therapy group (mean: 18.4 days versus 28.9 days, respectively; $\mathrm{p}=0.001$ ).

In the study by Driver et al. ${ }^{23}$ wounds were measured weekly, wound tissue biopsies obtained and wound fluid collected. At week four, average wound size
Fig 1. PRISMA flow diagram for study selection

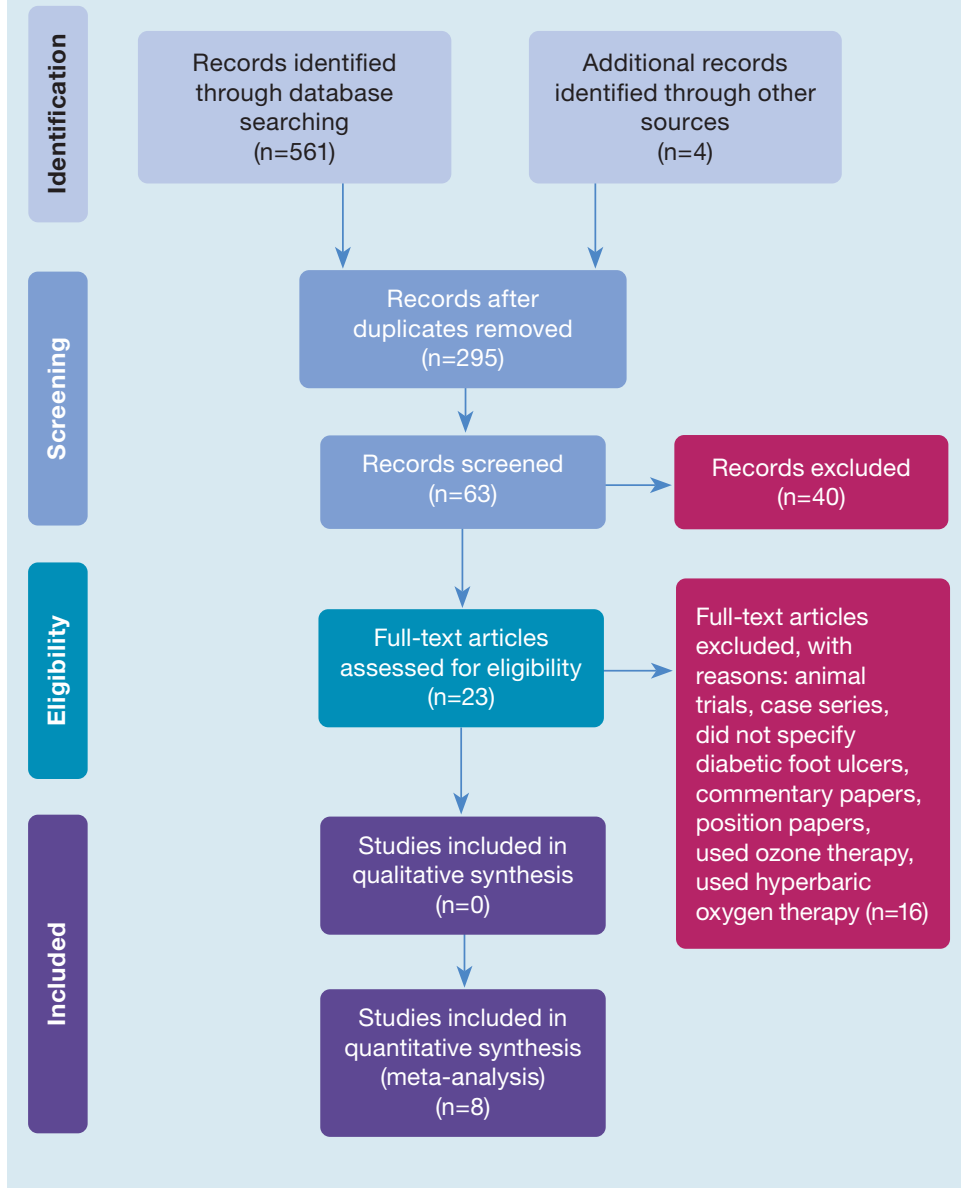

reduction was $87 \%$ (range: $55.7-100 \%$ ) in the treatment group compared with $46 \%$ (range: 15-99\%) in the control group $(\mathrm{p}<0.05)$. Changes in cytokine levels (IL-6, IL-8) and proteinases (MMP-1,-2,-9, TIMP-1) in

Fig 2. Forest plot of healing oxygen versus standard care

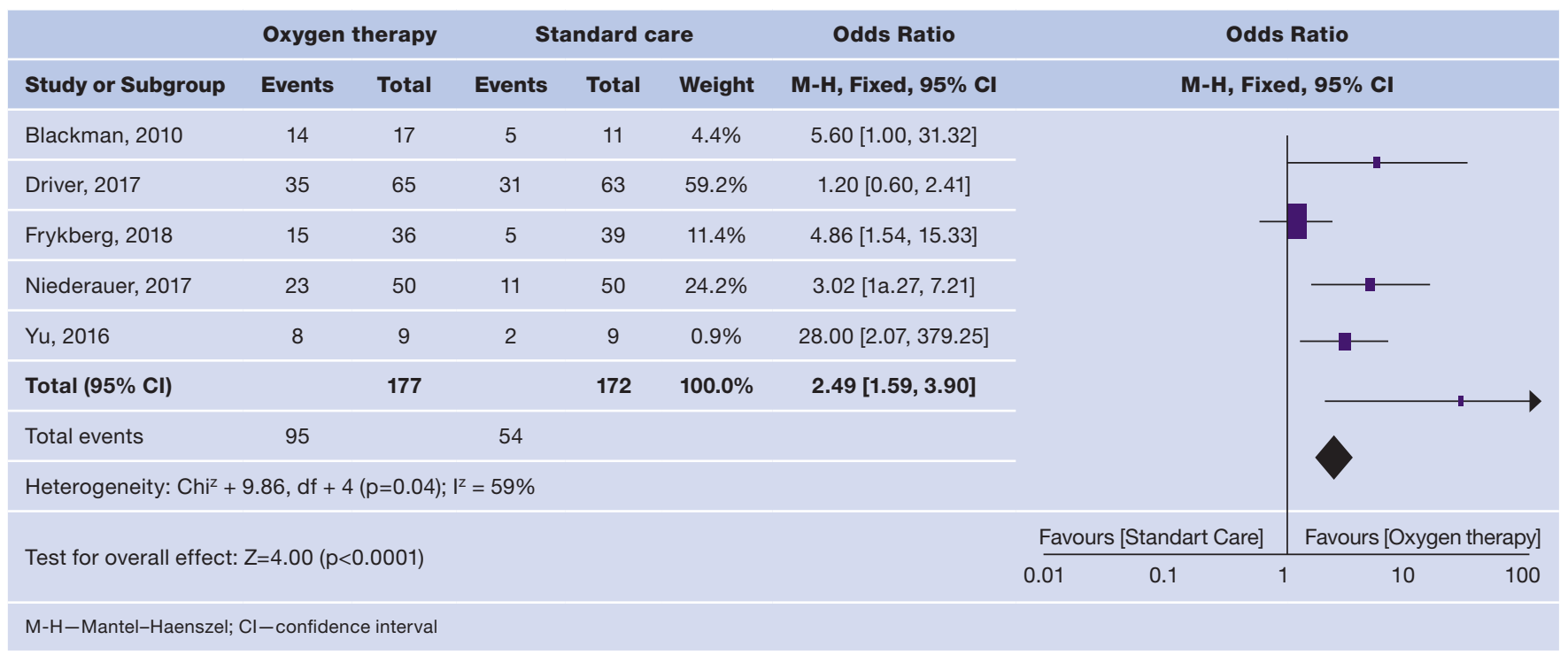


the wound fluid at weeks $2-4$ correlated with clinical findings. CD68+ macrophage counts showed a statistically significant reduction in response to the TOT group compared with the control group $(\mathrm{p}<0.01)$.

Recurrence rates: Blackman et al. ${ }^{18}$ compared DFU recurrence rates after 24 months in both the therapy group and the control group. They found there were no recurrences at the healed ulcer site in either the therapy group or control group. Hayes et al. ${ }^{22}$ stated in their study that there was no recurrence of any ulcers at follow-up.

In the trial by Niederauer et al., ${ }^{21}$ recurrence rates were collected at follow-up at 12 weeks. No statistical difference in the results was observed, with $87.5 \%$ in the therapy group remaining closed and $90 \%$ remaining closed in the placebo group. Frykberg et al. ${ }^{13}$ did not, as at the time of writing, report reoccurrence rates or time to heal figures.

Adverse events, ease of use and pain: Blackman et al. ${ }^{18}$ stated that no treatment-related adverse events were documented in either group. Hayes et al. ${ }^{22}$ provided feedback from patients regarding ease of use. The device was well tolerated and patients enjoyed being involved in their treatment. Pain levels were reduced: from 39\% of participants recording pain at $\geq 5 / 10$ at the start of the study to only $7 \%$ of participants with levels $\geq 5 / 10$ at the end of the study.

Driver et $a .^{23}$ found that there were fewer infections in the TOT group (3.5\%) compared with the control group (10.15\%), but the differences were not statistically significant. In addition, the authors reported that cellulitis incidents were more frequent in the control group $(6.9 \%)$ compared with the TOT group (1.2\%), but again, these differences were not significant.

\section{Quality appraisal}

The EBL Appraisal checklist was used to assess the methodological quality of the included studies in this systematic review by focusing on the four main domains: population, data collection, study designs and results. The assessment of these domains is summarised in Table 1, where validity figures can be found as well as any not reported, or unclear issues identified in each domain.

The mean validity score for all studies was $65.5 \pm 26.9 \%$. The minimum score was $0 \%{ }^{19}$ while the highest overall validity was $92 \% .{ }^{24}$ As can be seen in Table $1,37.5 \%$ of the studies scored $\geq 75 \%$, indicating that these studies were considered valid.

\section{Discussion}

The main goal of this systematic review was to examine the effect of TOT on healing of DFUs. All of the studies included produced positive results in favour of using TOT in the treatment of people with DFUs. It should be noted that the TOT was delivered by different methods. However, analysis showed that by using TOT, a DFU was $>2$ times more likely to heal than with standard care alone.

Primary outcomes measured number of wounds that gained wound closure or reduced wound circumference. Secondary outcomes were healing rates, recurrence rates, pain and device satisfaction; one paper looked at changes in biological markers in tissue samples of patients receiving therapy. In most of the studies reviewed, all the wounds were treated with standard care, regular debridement and offloading along with the TOT. It was interesting that three of the studies ${ }^{10,18,20}$ removed the possibility of wounds healing with standard care alone — without TOT_-by having a run-in treatment before commencing TOT.

In 2005, the Undersea and Hyperbaric Medical Society published a position statement on TOT for hard-to-heal wounds. ${ }^{25}$ The aim was to ensure that TOT was not called hyperbaric oxygen therapy and that studies referencing systematic hyperbaric oxygen therapy should not be used when discussing the physiology of TOT. The paper concluded by stating that TOT should be considered to have mixed results rather than better results than hyperbaric oxygen therapy.

There have been a number of reviews of TOT in wound care, all of which discussed the different delivery methods. ${ }^{11,12,26-28}$ Our review focused specifically on TOT and its effectiveness in patients with DFUs.

DFUs are complex wounds and markers of comorbidities and serious disease. ${ }^{29}$ A study by Mavrogenis et al., ${ }^{30}$ estimated that $50 \%$ of DFUs would become infected and of these infected DFUs $20 \%$ would require amputation.

Previous literature with regard to TOT was limited to case reviews, making clinicians wary about trying this new therapy. Our review of eight studies that incorporated 349 participants provides a meta-analysis from four trials and has given a narrative analysis of the remaining three studies. Based on our findings, TOT-whether in the form of continuous delivery of oxygen or as topical pressurised oxygen-may, when used as an adjunct to standard care, improve healing rates in patients with DFUs. It shows that TOT has the potential to positively impact on the effectiveness of wound healing with the potential to improve limb salvage and a patient's quality of life.

\section{Limitations}

In the search strategy of this systematic review the author focused on studies written in English only as there were no means to translate any studies in other languages. This may have resulted in language bias with perhaps important studies being missed that may have brought further evidence for or against the intervention.

There are also some limitations to the conclusions drawn. Only two of the eight studies included were deemed valid, ${ }^{10,21}$ and one study ${ }^{19}$ received a score of $0 \%$; the main weakness of the papers being sample size. Too small a sample size will result in imprecise estimates in a descriptive study and will fail to achieve a statistical 
significance in a comparative study. ${ }^{31}$ Also, recurrence rates were only measured in four of the eight papers. This would have been an important secondary outcome for all papers as a patient with a healed DFU has a $17-60 \%$ chance of another one occurring. ${ }^{32}$

\section{Recommendations for practice and research}

This systematic review has shown that there are few RCTs conducted to show the benefit of TOT. Following a robust search, only five RCTs were found. Further studies need to address follow-up care, patient quality of life and cost. More robust research is needed to highlight its possible benefit in the adjunct treatment of DFUs.

\section{Conclusion}

DFUs are a challenging area of wound care practice. For the patient they can have an impact on their morbidity and mortality. The high rate of amputations following infection of DFUs is costly to the patient and the health service. This review found evidence that the adjunct therapy of TOT may lead to faster healing rates and wound closure for some patients if used alongside standard diabetic foot care. Jwc

\section{References}

1 Lavery LA, Peters EJ, Armstrong DG et al. Risk factors for developing osteomyelitis in patients with diabetic foot wounds. Diabetes Res Clin Pract 2009; 83(3):347-352. https://doi.org/10.1016/j.diabres.2008.11.030 2 Smith D, Cullen MJ, Nolan JJ. The cost of managing diabetic foot ulceration in an Irish hospital. Ir J Med Sci 2004; 173(2):89-92. https://doi. org/10.1007/BF02914564

3 Boulton AJ, Vileikyte L, Ragnarson-Tennvall G, Apelqvist J. The global burden of diabetic foot disease. Lancet 2005; 366(9498):1719-1724. https://doi.org/10.1016/S0140-6736(05)67698-2

4 Bakker K, Apelqvist J, Lipsky BA et al; International Working Group on the Diabetic Foot. The 2015 IWGDF guidance documents on prevention and management of foot problems in diabetes: development of an evidence-based global consensus. Diabetes Metab Res Rev 2016; 32(32 Suppl 1):2-6. https://doi.org/10.1002/dmrr.2694

5 Buckley CM, O'Farrell A, Canavan RJ et al. Trends in the incidence of lower extremity amputations in people with and without diabetes over a five-year period in the Republic of Ireland. PLoS One 2012; 7(7):e41492. https://doi.org/10.1371/journal.pone.0041492

6 Cho NH, Shaw JE, Karuranga S et al. IDF Diabetes Atlas: global estimates of diabetes prevalence for 2017 and projections for 2045. Diabetes Res Clin Pract 2018; 138(138):271-281. https://doi.org/10.1016/j. diabres.2018.02.023

7 International Diabetes Federation (IDF). IDF Diabetes Atlas 8th Edition 2017. https://tinyurl.com/52rauzkr (accessed 15 July 2021)

8 Diabetes UK. Diabetes prevalence 2018. https://tinyurl.com/zmb3vwjy (accessed 15 July 2021)

9 Skerritt L, Moore Z. The prevalence, aetiology and management of wounds in a community care area in Ireland. Br J Community Nurs 2014; 19(Sup6 Suppl):S11-S17. https://doi.org/10.12968/bjcn.2014.19.Sup6.S11 $10 \mathrm{Yu}$ J, Lu S, McLaren AM et al. Topical oxygen therapy results in complete wound healing in diabetic foot ulcers. Wound Repair Regen 2016; 24(6):1066-1072. https://doi.org/ 10.1111/wrr.12490. 11 Gottrup F, Dissemond J, Baines C et al. Use of oxygen therapies in wound healing. J Wound Care 2017; 26 Sup5:S1-S43. https://doi. org/10.12968/jowc.2017.26.Sup5.S1

12 Howard MA, Asmis R, Evans KK, Mustoe TA. Oxygen and wound care: a review of current therapeutic modalities and future direction. Wound Repair Regen 2013; 21(4):503-511. https://doi.org/10.1111/wrr.12069. 13 Frykberg RG, Franks PJ, Edmonds $M$ et al. A multinational, multicenter, randomized, double-blinded, placebo-controlled trial to evaluate the efficacy of cyclical topical wound oxygen therapy (TWO2) in the treatment of chronic diabetic foot ulcers: the TWO2 Study. Diabetes Care 2018; 2019: https://doi.org/10.2337/dc19-0476

14 Glynn L. A critical appraisal tool for library and information research. Libr Hi Tech 2006; 24(3):387-399. https://doi.

org/10.1108/07378830610692154

15 Review Manager R. The Nordic Cochrane Centre, The Cochrane Collaboration. Version 5.3. ed. Copenhagen: The Nordic Cochrane Centre: The Cochrane Collaboration, 2014. https://tinyurl.com/4mns3un3 (accessed 27 September 2021)

16 Higgins J, Green S. Cochrane handbook for systematic reviews of interventions Version 5.1.0 [updated March 2011]. The Cochrane

Collaboration, 2011. https://handbook-5-1.cochrane.org/ (accessed 15 July 2021)

17 Copeland K, Purvis AR. A retrospective chart review of chronic wound patients treated with topical oxygen therapy. Adv Wound Care 2017; 6(5):143-152. https://doi.org/10.1089/wound.2017.0729

18 Blackman E, Moore C, Hyatt J et al. Topical wound oxygen therapy in the treatment of severe diabetic foot ulcers: a prospective controlled study. Ostomy Wound Manage 2010; 56(6):24-31
19 Kemp D, Hermans M. An evaluation of the efficacy of transdermal continuous oxygen therapy in patients with recalcitrant diabetic foot ulcer. J Diabet Foot Complicat 2011; 3(1):6-12

20 Driver VR, Yao M, Kantarci A et al. A prospective, randomized clinical study evaluating the effect of transdermal continuous oxygen therapy on biological processes and foot ulcer healing in persons with diabetes mellitus. Ostomy Wound Manage 2013; 59(11):19-26

21 Niederauer MQ, Michalek JE, Armstrong DG. A prospective, randomized, double-blind multicenter study comparing continuous diffusion of oxygen therapy to sham therapy in the treatment of diabetic foot ulcers. J Diabetes Sci Technol 2017; 11(5):883-891. https://doi. org/10.1177/1932296817695574

22 Hayes PD, Alzuhir N, Curran G, Loftus IM. Topical oxygen therapy promotes the healing of chronic diabetic foot ulcers: a pilot study. J Wound Care 2017; 26(11):652-660. https://doi.org/10.12968/ jowc.2017.26.11.652

23 Driver VR, Reyzelman A, Kawalec J, French M. A prospective, randomized, blinded, controlled trial comparing transdermal continuous oxygen delivery to moist wound therapy for the treatment of diabetic foot ulcers. Ostomy Wound Manage 2017; 63(4):12-28

24 Kemppainen BW, Urry DW, Swaim SF et al. Bioelastic membranes for topical application of a thromboxane synthetase inhibitor for protection of skin from pressure injury: a preliminary study. Wound Repair Regen 2004; 12(4):453-460. https://doi.org/10.1111/j.1067-1927.2004.12410.x. 25 Feldmeier JJ, Hopf HW, Warriner RA 3rd et al. UHMS position statement: topical oxygen for chronic wounds. Undersea Hyperb Med 2005; 32(3):157-168

26 Orsted HL, Poulson R, Baum J et al.; Advisory Group. Evidence-based practice standards for the use of topical pressurised oxygen therapy. Int Wound J 2012; 9(3):271-284. https://doi. org/10.1111/j.1742-481X.2012.00956.x

27 Dissemond J, Kröger K, Storck M et al. Topical oxygen wound therapies for chronic wounds: a review. J Wound Care 2015; 24 (2):53-54, 56-60, 62-63. https://doi.org/10.12968/jowc.2015.24.2.53

28 Sayadi LR, Banyard DA, Ziegler ME et al. Topical oxygen therapy and micro/nanobubbles: a new modality for tissue oxygen delivery. Int Wound J 2018; 15(3):363-374. https://doi.org/10.1111/iwj.12873

29 Kaufman H, Gurevich M, Tamir E et al. Topical oxygen therapy stimulates healing in difficult, chronic wounds: a tertiary centre experience. J Wound Care 2018; 27(7):426-433. https://doi.org/10.12968/

jowc.2018.27.7.426

30 Mavrogenis AF, Megaloikonomos PD, Antoniadou T et al. Current concepts for the evaluation and management of diabetic foot ulcers. EFORT Open Rev 2018; 3(9):513-525. https://doi.

org/10.1302/2058-5241.3.180010

31 Weber EJ, Hoo ZH. Why sample size estimates? Emerg Med J 2018; 35(12):755-756. https://doi.org/10.1136/emermed-2018-207763 32 Armstrong DG, Boulton AJ, Bus SA. Diabetic foot ulcers and their recurrence. N Engl J Med 2017; 376(24):2367-2375. https://doi. org/10.1056/NEJMra1615439

Reflective questions

- What are the challenges to healing a diabetic wound, and why?

- What is the role of topical oxygen therapy on diabetic foot ulcer healing rates?

- What is the role of topical oxygen therapy on diabetic foot ulcer recurrence rates? 